\title{
Effect of Network Infrastructure Factors on Information System Risk Judgments
}

\author{
Jennifer A. Cowley ${ }^{a}$, Frank L. Greitzer ${ }^{b, 1}$, Bronwyn Woods ${ }^{a}$ \\ ${ }^{a}$ Carnegie Mellon University/Software Engineering Institute, Pittsburgh, PA 15213, USA \\ ${ }^{b}$ PsyberAnalytix, Richland, WA 99352, USA
}

\begin{abstract}
Little is known about how perceived network topology factors, which are common components of information system risk metrics, impact human judgments of risk. Using a halffractional factorial design, this study experimentally manipulated five perceivable network topology factors (network partitioning, network diversity, wireless status, network footprint and connectivity) to assess the relationship between these factors and network risk judgments. The consistency of network risk ratings and rankings were evaluated for each of the 16 network topologies across a sample of 55 network security professionals who reviewed these topologies. Three robust significant main effects (network partitioning, wireless status, and connectivity) and one significant interaction (network partitioning $\mathrm{X}$ wireless status) were found. While some topologies were consistently rated and ranked as significantly more risky than others, there was some variability in ratings at each main effect level as well as the spread of the mean ratings between the two main effect levels (e.g., wireless and wired). We discuss the implications of our findings with respect to network risk metric rigor.
\end{abstract}

Keywords: network security; risk perception; risk judgment; information system risk; vulnerability; security metric; network topology; network risk assessment; risk management.

\section{Introduction}

Risk to information systems, which includes network risk, arises from threats to information systems that can cause the loss of data confidentiality, integrity, or availability and includes the adverse impacts to organizational operations (e.g., mission, functions, image, or reputation), organizational assets, individuals, or other organizations (NIST SP 800-53, 2013). The measurement of network risk in particular is important for a variety of reasons. First, networks are integral to communications between computers in an information system, thus they enable access to critical vulnerabilities that attackers seek to exploit. Effort spent to reduce risks through secure network configuration can limit access to those vulnerabilities, thus reducing overall system risks. Second, the measurement of network risk is important because organizations use

\footnotetext{
${ }^{1}$ Corresponding author. Tel: $+1509539-4250$

Contact information: jcowley@cert.org, Tel: +1 412 268-4461 (J. Cowley); Frank@PsyberAnalytix.com (F.L. Greitzer)
} 
risk metrics to prioritize how to spend their limited resources to secure their information systems (Elky, 2006; NIST, 2012). In current practice, an organization's network risk can be assessed with any of these metrics: Operationally Critical Threat, Asset, and Vulnerability Evaluation (OCTAVE) (Alberts \& Dorofee, 2001); NIST SP 800-30Rev1 (NIST, 2012) and the NIST CVSS V2.10 network risk measure; CORAS (Aagedal, Den Braber, Dimitrakos, Gran, Raptis \& Stolen, 2002); and Risk Management Framework (RMF) (Verdon \& McGraw, 2004).

Rigorous risk metrics are difficult to validate because they depend upon the composition of human perceptions of physical elements (e.g., computers, software, firewalls, etc.) and nonphysical abstractions (e.g., adversary type, subjective security levels, etc.). We identified two critical issues that must be addressed to improve the rigor of network risk metrics in light of these challenges. First, neither the risk research community nor network security practitioners have converged on an empirically validated definition of network risk (Renn, 1998) involving the objective and subjective components of network risk. Consequently, if practitioners are not using the same definition of risk, then miscommunications results (Fischhoff, 2009) that leads to confusion, incorrect assumptions or inaction. Second, the subjective component of network risk metrics (e.g., the NIST CVSS V2.10), introduces variance in human risk judgment. If these metrics include factors (e.g., availability, integrity, etc.) with high disagreement about the risk level of those factors, then the metric's underling model may have too much variance and suffer reliability and validity reductions. Due to the lack of published research on metric generation and validation for network risk metrics, it is unclear how much variability in the underlying metric model can be attributed to human judgments of subjective factors, such as availability. In addition to human perceptions of the network environment, other factors also impact human judgment such as memory lapses, personal experiences, attitudes and opinions, and perceptual biases (e.g., Pamula, Jammann, Jajodia \& Swarup, 2006; Gilovich, Griffin \& Kahneman, 2002).

To address these issues, we conducted an empirical investigation of the impacts of experimentally manipulated network topology factors (network partitioning, network diversity, wireless status, network footprint and connectivity) on expert judgments of network risk. Through both exploratory and quantitative analyses of risk rating and ranking data, we sought to identify those factors that were important for network risk judgments and to assess the variability among expert ratings and rankings of network risk. This study therefore examined characteristics that are potentially important to consider in any model of network risk and which should inform efforts to standardize subjective ratings used in network risk assessment.

This paper is organized as follows: Section 2, reviews related research and theory on the challenges of network risk metrics; Section 3, presents the research questions; in Section 4, we present the experimental method and procedure, including the participant sampling and study materials used; Section 5 reports the results of exploratory and statistical modeling of the data obtained; and finally Section 6 discusses the results, implications and limitations of the study, and possible future research. 


\section{Related Work}

In this section, we first review the phenomenology of risk before we review research from the social sciences on how perception can influence judgment as it pertains to risk, and how the measurement of judgments is challenging.

Risk is a socially constructed phenomenon (Kukla, 2000): its meaning and how it is represented is based on a degree of agreement among the parties affected, each of whom has the ability to modify and evolve this meaning over time. For example, people have different opinions of what happiness is and how it is visually represented. In contrast, physical height is not socially constructed; its measurement does not entail the subjective interpretation of what height is; however, the interpretation of tall vs. short is socially constructed and is relative to individual experiences. Because happiness is identified through subjective interpretation of visual cues that differs across group cultures (Diener, 2000), no single representation of happiness theoretically exists. The class of psychometrics that measure socially constructed phenomena is not designed to reduce the importance of the subjective judgments; the subjective judgment is the foundation of this class of psychometrics. Like happiness, the socially constructed concept of network risk is likely based on idiosyncratic interpretations of what network risk is and how it is identified. Yet, network risk publications (Longstaff, Chittister, Pethia \& Haimes, 2000; Pamula et al., 2006) often try to minimize the subjective component of risk metrics to improve their objectivity. This impacts metric validity because the concept of risk is based on group consensus. Therefore, we question the validity of network risk metrics that are designed from a single person's (the metric designer) vantage point given that one this single view point may run counter to the perspectives of the population of network risk professionals. Our research approach exclusively focuses on the subjective interpretation of network risk in order to understand how network risk is socially constructed.

Prior research identified the term risk as a psychological construct (Cronbach \& Meehl, 1955), typically represented by the aggregation of dimensions (e.g., likelihood of adversarial penetration, impact of a lack of network resilience, etc.). Arguably, dimensions could be considered types of risk (e.g., financial risk, availability risk, competing-mission risk, etc.) but grounded analysis research is required on human network risk judgment to determine the degree of orthogonally between dimensions and types of risk. Furthermore, dimensions are based on perceived, tangible cues in the network environment that we call factors (e.g., zero redundant connections to the internet). We conjecture that a relationship exists between a person's definition of network risk, their own dimensions that they believe are important to risk, and the factors that they perceive and consider for risk judgment. To our knowledge, there is no agreement about how the term risk is defined and what dimensions constitute risk (Bauer, 1960; Crespo, del bosque \& de los Salmones Sanchez, 2009; Dowling, 1986; Fischhoff, 2009; Gemünden, 1985; Haimes, 2009; Ingene \& Hughes, 1985; Ross, 1975). The term network risk 
suffers the same lack of agreement (Renn, 1998). We know from prior research that network risk judgments are based on perceptions of network vulnerabilities, threats (e.g., adversaries, natural disasters, etc.), asset importance, resilience, and counter measures (Alberts \& Dorofee, 2001; Ghandi \& Lee, 2011; see also DOD Instruction 8500.2 available at, http://fas.org/irp/doddir/dod/d8500_2.pdf). However, we do not know the degree of consensus about the importance of these perceptions for network risk judgments.

One way to validate risk metrics is to assess concurrent validity or how well the metric outcomes predict subsequent adverse network events. However, understanding what exactly is occurring on the network at any given moment, also called ground truth, is unattainable. Currently, we are experiencing a shortage of tools and technology that quickly and accurately identifies whether a malicious actor is on a given network at any point in time. Hence, it is impractical to use concurrent validity to assess metric validity. In the absence of ground truth on network activities, researchers can use group consensus of subject matter expert (SME) judgments as a proxy for ground truth (Buchanan, Davis, \& Feigenbaum, 2006).This consensus can assist in both the identification of adversarial activity and the interpretation of these activities with respect to network risk. Our research begins to evaluate group consensus on network risk judgments based on what is perceived in the network.

We cannot ignore the corpus of research from the social sciences that illustrates the unreliability of human perception and judgment. To clarify some terminology distinctions in the research literature, human information processing (HIP) theories (Wickens \& Carswell, 1997; Cowan, 1997) delineate risk perception, risk judgment (also called decision making), risk attitudes (Weber, Blais \& Betz, 2002) and risk behaviors (Eagly \& Chaiken, 1993; Ajzen \& Fishbein, 1977; Ajzen, 1991). Different research efforts focus on each of these HIP components but they are not semantically identical. Our research effort focuses exclusively on risk perception and risk judgment. From the foundational literature on basic human visual perception, we understand that perception is contingent upon the ability to discern signal (also called cues) from noise. The perceived cues are then processed and a judgment is made about what is being viewed. Because network risk is not a tangible phenomenon to perceive, we reviewed how people perceive abstractions. Several visual perception studies involving the perception of visual abstractions (Boring, 1930; Dallenbach, 1951; Gauthier \& Tarr, 1997; Miller, 1999; Tarr, 2000) found that different people perceive different objects in abstractions based on patterns of cues embedded in noisy contexts (for reasons why, see Sternberg, 2008, Ch. 4). We also understand that cognitive processing of cue patterns can involve the human's manipulation of perceived cues such that the memory of such cue patterns becomes a distorted representation of reality (Edelman \& Weinshall, 1991; Peterson, 1999; Tarr, 1995). Therefore, humans can unintentionally distort perceptions of subjective and objective reality. To drive home the point, humans can manipulate, misperceive or fail to perceive relevant cues in different contexts, and people can change the way they perceive, judge and remember reality based on their own perceptual viewpoints. Even though these studies involved visual perception and judgment of objects, some of these findings 
may be applicable to the study of perceivable network factors that impact the variability of network risk judgments, since much of the information gleaned to make those judgments is visual. To build on that foundational research, we know that risk perception can be influenced by context, defined as the perceivable aspects physically and temporally surrounding an event or circumstance (Fischhoff, Detels, Beaglehole, Lansing, \& Guilliford, 2009; Poulton, 1989). In our study, we evaluate the change in risk judgments when perceivable visual network factors change.

\section{Research questions}

Throughout this manuscript, we use the NIST CVSS V2.10 to illustrate the complexity of network risk metrics from a social sciences perspective. We chose this metric because it is recommended to all U.S. government agencies as part of NIST's statutory responsibility under the Federal Information Security Management Act of 2002 (44 U.S.C. § 3541). The factors used in our research study were based on prior research findings from our lab, which were subsequently compared to those listed in the NIST CVSs V2.10 metric. Cowley, Greitzer \& Woods (2015) conducted surveys and focus groups to identify, among network professionals, what network risk factors were important to network risk judgments. The resultant factors that could be depicted together in network topologies include: 1) network disposition, which is whether a single machine on the network can access both the Internet and the Intranet at any point in time; 2) network characteristics, which is whether or not there is diversity in the type of platform or operating systems employed in the network; 3) wireless status, which is whether the network includes wireless communications; 4) footprint, which is whether the network architecture is centrally-managed or distributed; and 5) connectivity, which is the degree of connectivity of the organization's network to the Internet. These factors also relate to different components of the NIST CVSS V2.10 network risk metric, which divides the metric into three sub-metrics-base metrics, temporal metrics, and environmental metrics. In practice, as described in the NIST report, A Complete Guide to the Common Vulnerability Scoring System Version 2.0, “...the base and temporal metrics are specified by vulnerability bulletin analysts, security product vendors, or application vendors because they typically have better information about the characteristics of a vulnerability than do users." (Mell, Scarfone, \& Romanosky, 2007, p. 5). The environmental metrics are designed to reflect the more specific organizational IT environment, and these inputs are provided by users (network security professionals within the enterprise), because they can best assess the vulnerability's potential impact within their own IT environments (Mell et al., 2007). Our chosen topological factors for our research fit under the class of IT environment category of the NIST CVSS V2.10 network risk metric. We adopted binary values for these factors, described as follows:

- Network partitioning (network disposition) - partitioned vs. non-partitioned. A strategy to reduce the potential impact of network attacks is virtualization and partitioning of network resources. This isolation of network resources limits access to resources that are 
within a partition, so that systems in one partition cannot encroach on resources in a different partition. This prevents unauthorized access to resources and helps to prevent the spread of malware (Scarfone, Souppaya \& Hoffman, 2011).

- Network diversity (network characteristics)-homogeneous vs. heterogeneous. Homogeneity refers to use of a single protocol, software, or technology at each layer of the network's architecture. While homogeneous networks have improved interoperability and reduced costs compared to heterogeneous networks, they are more vulnerable to malicious attacks that exploit a single weakness common to all of its components. In a heterogeneous network where critical functionality is provided by a diverse set of protocols and implementations, attacks that focus on a weakness of one such protocol or implementation will not be able to bring down the entire network, even if some of its components are compromised (Zhang, Dao, Vin, Alvisi \& Lee, 2002).

- Wireless status-wireless vs. wired. All vulnerabilities associated with wired networks apply to wireless networks, but some are exacerbated by wireless connectivity and some new vulnerabilities are created. As noted in a NIST report on wireless network security, "Perhaps the most significant source of risks in wireless networks is that the technology's underlying communications medium, the airwave, is open to intruders, making it the logical equivalent of an Ethernet port in the parking lot (Karygiannis \& Owens, 2002, p. ES-1)".

- Network footprint-distributed vs. centrally managed. In a distributed communication network, each station is connected to all adjacent stations rather than to a few switching points, as in a centralized system. The advantage of a distributed configuration is its greater survivability in the face of enemy attacks directed against nodes, links or combinations of nodes and links (Baran, 1964).

- Connectivity-high vs. low connectivity. This term relates to the broader concept of network availability, which is defined as the ability of the network to stay operational in case of network element failures (Clemente, Bartoli, Bossi, D'orazio \& Cosmo, 2005). Availability can be inferred from the degree of connectivity to the internet service provider's network-i.e., whether or not the network includes redundant connections to the Internet.

The objectives of our research are to determine how the manipulation of perceived network factors impacts judgments as well as to what degree study participants agree on those judgments. Another way to depict group agreement or consensus is through the model fit of our collected data. More specifically, these objectives are captured in the following research questions (RQ):

RQ1: For the network infrastructure factors examined, which of these five factors and two-factor interactions significantly influenced network risk perception, and what was their impact? 
RQ2: How well does our statistical model generated for Q1 describe our data and in what ways does it fail? How much variability in human judgment of network risk remains after accounting for the impact of each factor?

\section{Materials and methods}

In this study, network professionals with varying expertise assessed the network risk levels of simple network topologies. Using a fractional factorial design, we systematically manipulated the combination of various factor levels, which we depicted in network topologies, and obtained aggregate judgments on the impact magnitude and direction of each factor and factor interaction with respect to network risk judgment. Since an individual's expertise might engender different perspectives on network risk, we assessed the variability between and within individuals. We recognize that measuring group consensus for each factor may actually be a measure of widespread heuristic decision making (Sjoberg, 2000) rather than an approximation of any ground truth perspective on network risk. Therefore, we focused on the empirical documentation of risk judgment consensus and not the accuracy of the group consensus. In general, each participant reviewed 16 different scenarios and provided an overall network risk rating (between 0 and 100) and a network risk ranking (low, medium, or high) for each. In this section, we review the experimental variables, participant sampling, study design, study materials and procedure.

\subsection{Experimental variables}

An objective of this research was to investigate the directional impact of five network infrastructure factors and their possible two-factor interactions on network risk judgments. Since text-based descriptions of networks can lead to different subjective interpretations of what is being described (Bleich, 1978; Dixon, Bortolussi, Twilley \& Leung, 1993; Ragland, 1978; Slatoff, 1970) we used network topology factors instead, which have historically been included in risk assessment models and tools (e.g., Phillips and Swiler, 1998; Jojodia, Noel, O'Berry 2005; Pamula, et al., 2006; Ahmed, Al-Shaer, \& Khan, 2008). We used a factor scenario method (Rossi, 1951) to depict these topologies which we elaborate further on in section 2.3.1. The factors depicted in our factor scenarios were network partitioning, network diversity, wireless status, network footprint, and connectivity, as defined in Section 1.2.

\subsection{Participants}

\subsubsection{Sampling}

The techniques to sample from our target population shifted over time. The target population defined in our sampling frame comprised individuals employed as network professionals (e.g., systems architects, network engineer, network defense analyst, network security analyst) who were skilled at reading and inferring meaning from network topologies. Prior to sampling, we 
believed that our target population was a relatively large population. Instead, we learned immediately after we began recruiting that our target population was a difficult to reach population. Therefore, we chose social science sampling methods for difficult to reach populations and attempted to address the statistical concerns of this class of sampling methods. Our sampling frame did not target subject matter experts (SMEs) since valid metrics indicating a person's level of expertise (novice, intermediate, advanced, etc.) on various technical competencies, were lacking. In addition, self-reports of expertise is not necessarily a viable alternative because reports on expertise-related demographic variables (e.g., the number of years of experience, job title, etc.) have been found to be poor indicators of a person's level of expertise (Ericsson \& Lehmann, 1996; Sosniak, 2006). Furthermore, we feared that participant exclusion based on 'level of expertise' might hamper chances of achieving adequate sample sizes from a relatively small population. In lieu of SMEs, our sample frame targeted individuals from a variety of different job roles at different levels of expertise to improve the chances of obtaining a healthy sample size. We ultimately resorted to the use of snowball sampling which is a method commonly used when sampling requires the knowledge and assistance of insiders to complete the sampling (Biernacki \& Waldorf, 1981). Our sample is therefore potentially biased, though we have no reason to believe that the snowball sampling procedure favors individuals with particular interpretations of our network factors. We do not believe that the sampling bias impacted our conclusions substantially, but of course this must remain a concern when interpreting our findings. Future studies should take care to design sampling schemes that may work better for this population.

To solicit participants, we first purchased a marketing list of cybersecurity professionals from Gartner and between March 26, 2013 and April 15, 2103 we used email invitations to invite all 3,500 contacts to participate in our study. We received only two completed records from this approach. Next, we used a snowball convenience sampling strategy (Biernacki \& Waldorf, 1981) from April 1, 2013 to June 14, 2013, starting with our organization's network architects who disseminated the study participation invitations to their colleagues. This sampling produced a total of 55 complete records. The data from these 55 participants are the focus of the analytic efforts described below.

\subsubsection{Sample Demographics}

For the 55 participants who completed the survey, the mean number of years employed as network professionals was 11.5 ( $S D=8.6$ years). Most study participants worked in the areas of information technology, banking and finance, and academia. Table 1 indicates the types of business sectors represented in the sample (participants could select multiple sectors). 
Table 1. Sectors Supported by the Study Participants (Self-Reported, $n=55$, multiple selections allowed)

\begin{tabular}{ll}
\hline Sector & Frequency \\
\hline Information technology & 23 \\
Government & 12 \\
Military & 9 \\
Contractor & 9 \\
Banking/finance & 9 \\
Academia & 6 \\
Public health / Healthcare & 5 \\
Communication & 5 \\
Other & 5 \\
Energy & 3 \\
Training & 2 \\
Defense industrial base & 2 \\
Transportation systems & 1 \\
Postal and shipping & 1 \\
Nuclear reactors, materials, and waste & 1 \\
Critical manufacturing & 1 \\
Agriculture / food & 1 \\
\hline
\end{tabular}

For group consensus purposes, a group of varying levels of expertise on a wide variety of cybersecurity technical competencies would be ideal. Given a small sample size, a lack of metrics that clearly and accurately identify an individual's level of expertise on a technical competency, and a difficult to reach target population, we expected our sample to be less than ideal. We did find that there was some variability in job titles and self-reported expertise; the more variability the better. An assessment of self-reported job titles found that $72 \%$ were in operations, $15 \%$ of reported job titles were ambiguous (e.g., Consultant, computer scientist), 9\% were in leadership and personnel management positions, and $4 \%$ were in research. While it is difficult to infer job functions from job titles, the most frequent job title was network engineer ( 9 responses, $17 \%$ of sample) followed by network technician (4 responses, $7 \%$ of sample). Thus, there was a wide variety of non-redundant job titles, which provided minimal information on job 
functions. The self-reported job-related expertise yielded more clues as to what our participants were skilled at doing in their own jobs:

- Network engineer/architect: 24 participants (44\%) identified their own expertise in designing and configuring networks.

- Network maintenance and operations: 14 participants (25\%) identified their own expertise in day to day operations of the system, including routing, switching, and firewall operations.

- Network security/cybersecurity analyst: 12 participants (22\%) identified their own expertise in network security activities such as network forensics, penetration testing, red teaming, and intrusion detection.

- Software development/engineering: 7 participants (12\%) identified their own expertise in systems programming and software development.

- Leadership and management: 7 participants (12\%) identified their own expertise in IT management and planning, contingency planning, and project/product management.

- Other sparsely represented expertise included system administration/firewall administration (4\%) and incident response (malware/reverse engineer) (4\%).

\subsection{Study Design, Materials, and Procedure}

\subsubsection{Study Design}

A modified vignette-based factor scenario method (Rossi, 1951) from the social sciences was used to depict a fractional factorial experimental design. Vignette-based factor scenarios (Rossi, 1951) are used to study complex human judgments based on factors embedded in the scenario. In lieu of the traditional text-based vignettes to represent factor manipulations, we used pictorial representations of networks (network topologies). To assess which factors and two-factor interactions consistently were judged as high, medium and low levels of network risk, we used a fractional factorial design to display factor level combinations in network topologies for the following five factors discovered in preliminary research (Cowley, Greitzer \& Woods, 2015): network partitioning (they called this network disposition), network diversity (they called this network characteristics), wireless status, footprint, and connectivity (they called this network availability),. These five factors are described in Table 2. To avoid participant fatigue that would 
be associated with testing all $2^{5}=32$ combinations of the five binary factors, we used a half fractional-factorial design that targeted the assessment of all main effects and 2-factor interactions. Other multi-factor interactions were not of interest due to interpretation difficulties.

Table 2. Definition of Five Factors Used and Respective Levels

\begin{tabular}{|c|c|c|}
\hline Factor & Levels & Description \\
\hline $\begin{array}{l}\text { Network } \\
\text { Partitioning }\end{array}$ & $\begin{array}{l}\text { 0: Partitioned } \\
\text { 1: Not partitioned }\end{array}$ & $\begin{array}{l}\text { - A partitioned network means the subnets are } \\
\text { separated by a guard that exerts access control. } \\
\text { - A topology of a non-partitioned network has } \\
\text { switches (instead of guards) that connect the } \\
\text { subnets but do not exert access control. }\end{array}$ \\
\hline $\begin{array}{l}\text { Network } \\
\text { Diversity }\end{array}$ & $\begin{array}{l}\text { 0: Homogeneous } \\
\text { 1: Heterogeneous }\end{array}$ & $\begin{array}{l}\text { - A homogeneous network means all the computers } \\
\text { using the network are the same (all servers). } \\
\text { - A heterogeneous network means there is a roughly } \\
50 / 50 \text { mixture of servers and user terminals } \\
\text { connected to the network. }\end{array}$ \\
\hline $\begin{array}{l}\text { Wireless } \\
\text { Status }\end{array}$ & $\begin{array}{l}\text { 0: Not Wireless } \\
\text { 1: Wireless }\end{array}$ & $\begin{array}{l}\text { - A wireless network means the connection from the } \\
\text { switches/guards to the computers on the network is } \\
\text { wireless. } \\
\text { Otherwise, the connection is wired. } \\
\text { Note: In either case, the connections between the other } \\
\text { components (guards/switches to routers, routers to } \\
\text { internet) are wired. }\end{array}$ \\
\hline Footprint & $\begin{array}{l}\text { 0: Distributed } \\
\text { 1: Centrally Managed }\end{array}$ & $\begin{array}{l}\text { - If the architecture is distributed, there are two } \\
\text { distinct networks connected to the internet. Each } \\
\text { topology has one network operations center } \\
\text { (NOC). If the management is distributed, the NOC } \\
\text { must manage one network remotely, over the } \\
\text { internet. } \\
\text { - If the architecture is centrally managed, the NOC } \\
\text { does not have to send information over the internet } \\
\text { to manage any device because there is only one } \\
\text { network connected to the internet. }\end{array}$ \\
\hline Connectivity & $\begin{array}{l}0 \text { : high connectivity } \\
1 \text { : low connectivity }\end{array}$ & $\begin{array}{l}\text { - In a high-connectivity or available network, there is } \\
\text { a redundant connection from the network } \\
\text { (specifically, the border router) to the internet. In } \\
\text { the case of a distributed architecture, each network } \\
\text { has two connections to the internet. } \\
\text { - Otherwise, there is only one connection from the } \\
\text { router to the internet. } \\
\text { Note: In both cases, there is exactly one border router } \\
\text { per network; the number of wired connections to the } \\
\text { internet is what changes. }\end{array}$ \\
\hline
\end{tabular}

The resultant design, a $2^{5-1}$ Resolution $\mathrm{V}$ half-fractional-factorial experimental design (Montgomery, 2009, p. 297), reduced the number of experimental conditions from 32 to 16. Each experimental condition, which we call a scenario, had one network topology that reflected one of the sixteen permutations. Figure 1 provides a legend for interpreting all elements used in these 
network topologies depicted in each experimental condition. Figure 2 provides reduced-size representations of all 16 scenarios. Table 3 shows the design matrix giving the factor levels for each of the scenarios.

Table 3. Design Matrix indicating the 16 permutations used in the $2^{5-1}$ Resolution V Half-Fractional Factorial Design.

\begin{tabular}{|c|c|c|c|c|c|}
\hline Scenario & $\begin{array}{l}\text { Factor E: } \\
\text { Network } \\
\text { Partitioning }\end{array}$ & $\begin{array}{l}\text { Factor D: } \\
\text { Network } \\
\text { Diversity }\end{array}$ & $\begin{array}{l}\text { Factor C: } \\
\text { Wireless } \\
\text { Status }\end{array}$ & $\begin{array}{l}\text { Factor B: } \\
\text { Footprint }\end{array}$ & $\begin{array}{l}\text { Factor A: } \\
\text { Connectivity }\end{array}$ \\
\hline 1 & 0 & 0 & 1 & 1 & 1 \\
\hline 2 & 0 & 0 & 1 & 0 & 0 \\
\hline 3 & 1 & 0 & 1 & 1 & 0 \\
\hline 4 & 1 & 0 & 0 & 0 & 0 \\
\hline 5 & 1 & 0 & 0 & 1 & 1 \\
\hline 6 & 1 & 0 & 1 & 0 & 1 \\
\hline 7 & 0 & 0 & 0 & 1 & 0 \\
\hline 8 & 1 & 1 & 0 & 0 & 1 \\
\hline 9 & 1 & 1 & 1 & 0 & 0 \\
\hline 10 & 0 & 1 & 1 & 0 & 1 \\
\hline 11 & 1 & 1 & 1 & 1 & 1 \\
\hline 12 & 0 & 1 & 1 & 1 & 0 \\
\hline 13 & 1 & 1 & 0 & 1 & 0 \\
\hline 14 & 0 & 1 & 0 & 1 & 1 \\
\hline 15 & 0 & 1 & 0 & 0 & 0 \\
\hline 16 & 0 & 0 & 0 & 0 & 1 \\
\hline
\end{tabular}

In our scenario beta testing, we learned that text-based descriptions of various factor levels caused latent confounds to arise based on other non-perception aspects of human judgment (e.g., attitudes, memory biases, etc.) that negatively impacted network risk judgments. Therefore, we switched all text-based scenarios to pictorial representations (network topologies) for each of the 16 conditions. In addition, we recognized that the manipulations of factors are not always 
visually perceivable to our target population so we asked beta-test network architects to indicate what levels of each of the five factors were represented in each topology or scenario. We ensured our beta testers' perceptions matched our factor manipulation regime prior to launching the survey.

Key:
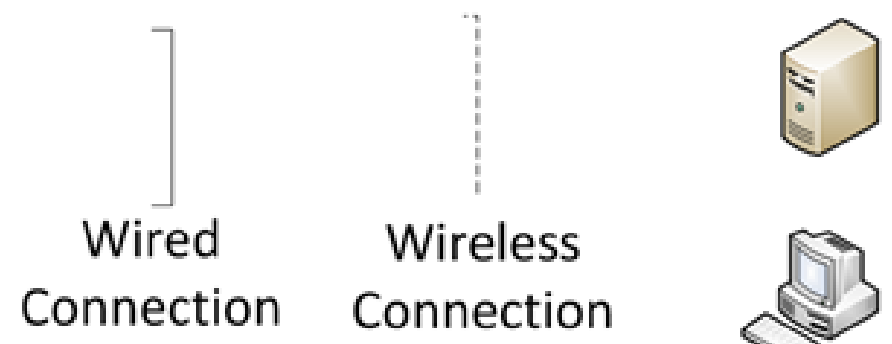

Server

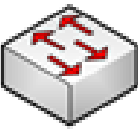

Switch

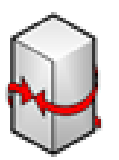

Guard

(Dynamic Firewall and IPS)
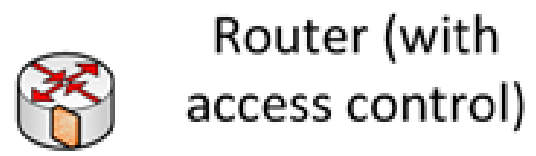

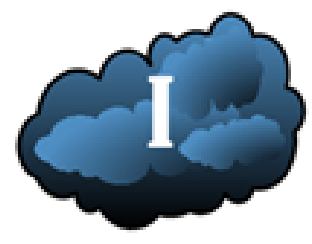

Internet
Client

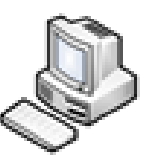

Network Operations

Center

Figure 1. Network Topology Legend 


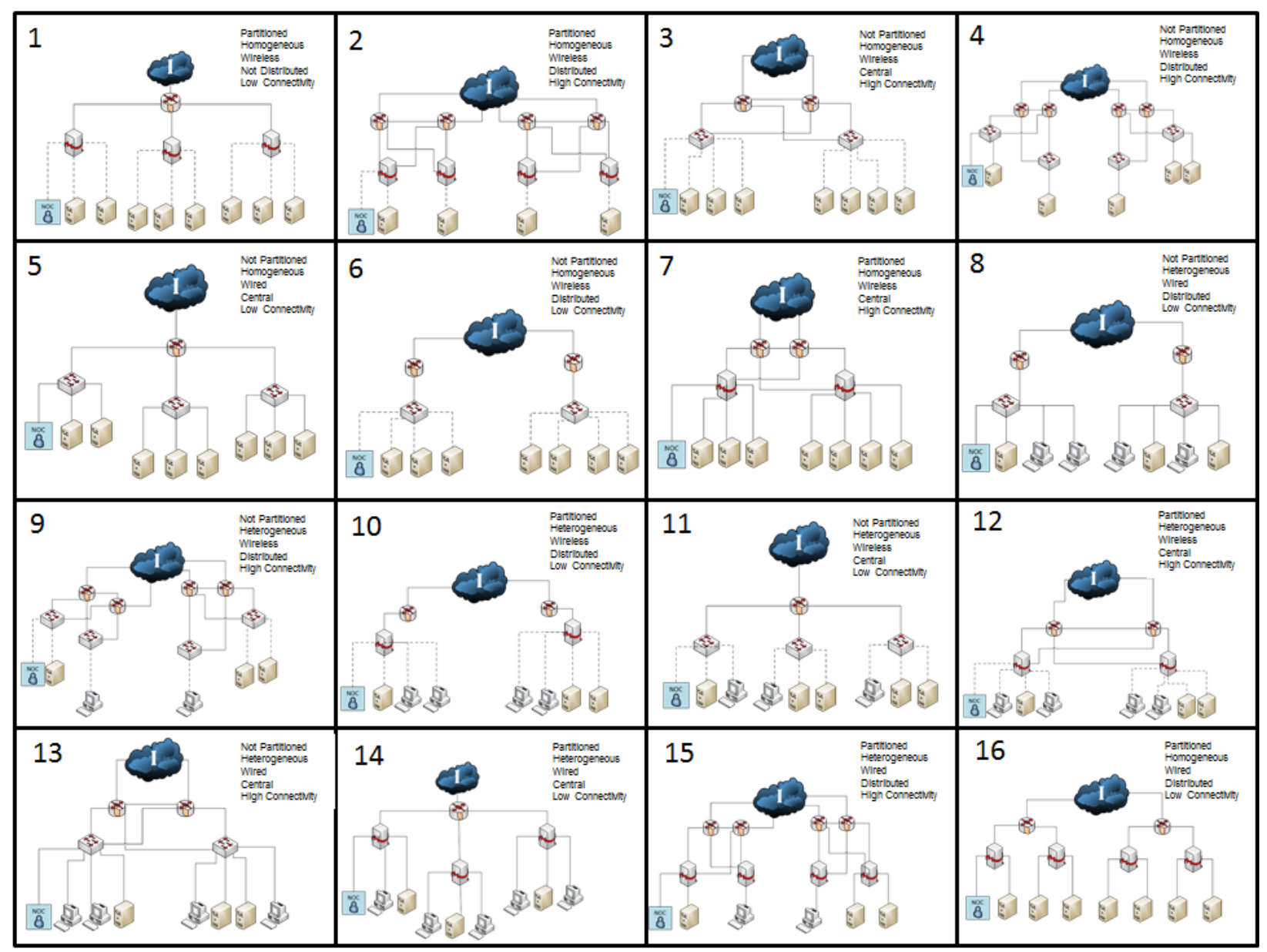

Figure 2. Network Topologies for Scenarios 1-16

\subsubsection{Materials and Procedure}

We used an online survey method hosted by SurveyGizmo ${ }^{\mathrm{TM}}$ to collect network risk judgments on each of the 16 resultant network topologies. The survey had three sections: informed consent, network topology scenarios, and demographics. After providing informed consent, each participant reviewed the 16 scenarios in a random order.

For each scenario in turn, participants provided two types of risk judgments. First, they assigned a low-medium-high ranking based on the standardized NIST network risk levels published in NIST SP800-30 (NIST 2012). We shall refer to this ordinal measure as NIST RANK, which has the following definitions based on the NIST guidance (NIST 2012, p. 23):

- High-Exercise of the vulnerability (1) may result in the highly costly loss of major tangible assets or resources; (2) may significantly violate, harm, or impede and organizations' mission; or (3) may result in human death or serious injury. 
- Medium-Exercise of the vulnerability (1) may result in the costly loss of tangible assets or resources; (2) may violate, harm, or impede an organization's mission, reputation, or interest; or (3) may result in human injury.

- Low-Exercise of the vulnerability (1) may result in the loss of some tangible assets or resources, or (2) may noticeably affect an organization's mission, reputation, or interest.

The second risk judgment was a measure of overall network risk in which participants were asked to assign a network risk rating between 0 and $100(0=$ not at all risky and $100=$ extremely risky). We refer to this measure as RISK RATING. To assign the rating, the participant adjusted a slider control on a digital visual analog scale with polar text anchors. The slider control included a numeric readout of the slider position in a pop-up window. By default, the slider was placed at the zero point of the scale underneath the "not at all risky" text anchor. Participants were required to adjust all sliders before moving onto the next question.

A demographics section at the end of the survey asked participants to indicate what entities they supported (military, government, independent contractor, and/or industry), whether they were an independent contractor, and which of 18 DHS business sectors they supported in their work (see https:/www.dhs.gov/news/2010/11/18/dhs-highlights-two-cybersecurityinitiatives-enhance-coordination-state-and-local). Respondents also indicated their job title and job-related expertise. To close out the survey, we debriefed participants and provided additional instructions on how to be financially compensated with a \$20 Amazon gift card.

\section{Results}

We conducted exploratory analyses and statistical modeling of the data obtained in this study. The exploratory analyses investigated within-subject and within-scenario evaluations of the responses to identify response patterns and eccentricities in the data. It is apparent that some participants did not interpret the task as intended (for instance, by giving the same rating to all scenarios citing a lack of available information to evaluate the impact of a potential attack). We also observed that participants used the available rating and ranking scales inconsistently. However, we did not observe consistent biases that would lead to spurious results. Since the results are extremely robust with respect to the impact of the network factors on perceived risk, we did not remove any suspicious data in our modeling analysis. If anything, removing participants such as the one described above would only strengthen our results.

The exploratory analysis examined the experts' consistency in assigning the network risk ratings at the scenario level (research question Q2). We assess research question 1 by fitting a linear model to the RISK RATINGS (scores between 0 and 100) and an ordered logistic regression to the NIST RANK data (low, medium or high risk). 


\subsection{Exploratory Data Analysis}

To understand participants' judgment consistency (the variability in interpretations of network risk level judgments by network professionals), we conducted an exploratory analysis of the distribution of RISK RATINGS for each participant (within-subjects analysis) and the distribution of participants' RISK RATINGS for each scenario (within-scenario analysis). In addition, we assessed the frequency distributions of NIST RANK data (high, medium and low risk) for each scenario.

Figure 3 depicts the data distributions across all 16 scenarios and all 55 participants, showing RISK RATINGS, NIST RANK data, and the relationship between the two. The X-axis is an index of the individual participants. For each participant, the sixteen plotted points reflect the scores for the sixteen scenarios. The position of the point on the $\mathrm{Y}$-axis indicates the RISK RATING score; the NIST RANK score is depicted using redundant coding with a black " $\mathrm{x}$ " = low risk, blue "o" = medium risk, and red "*” (asterisk) $=$ high risk.

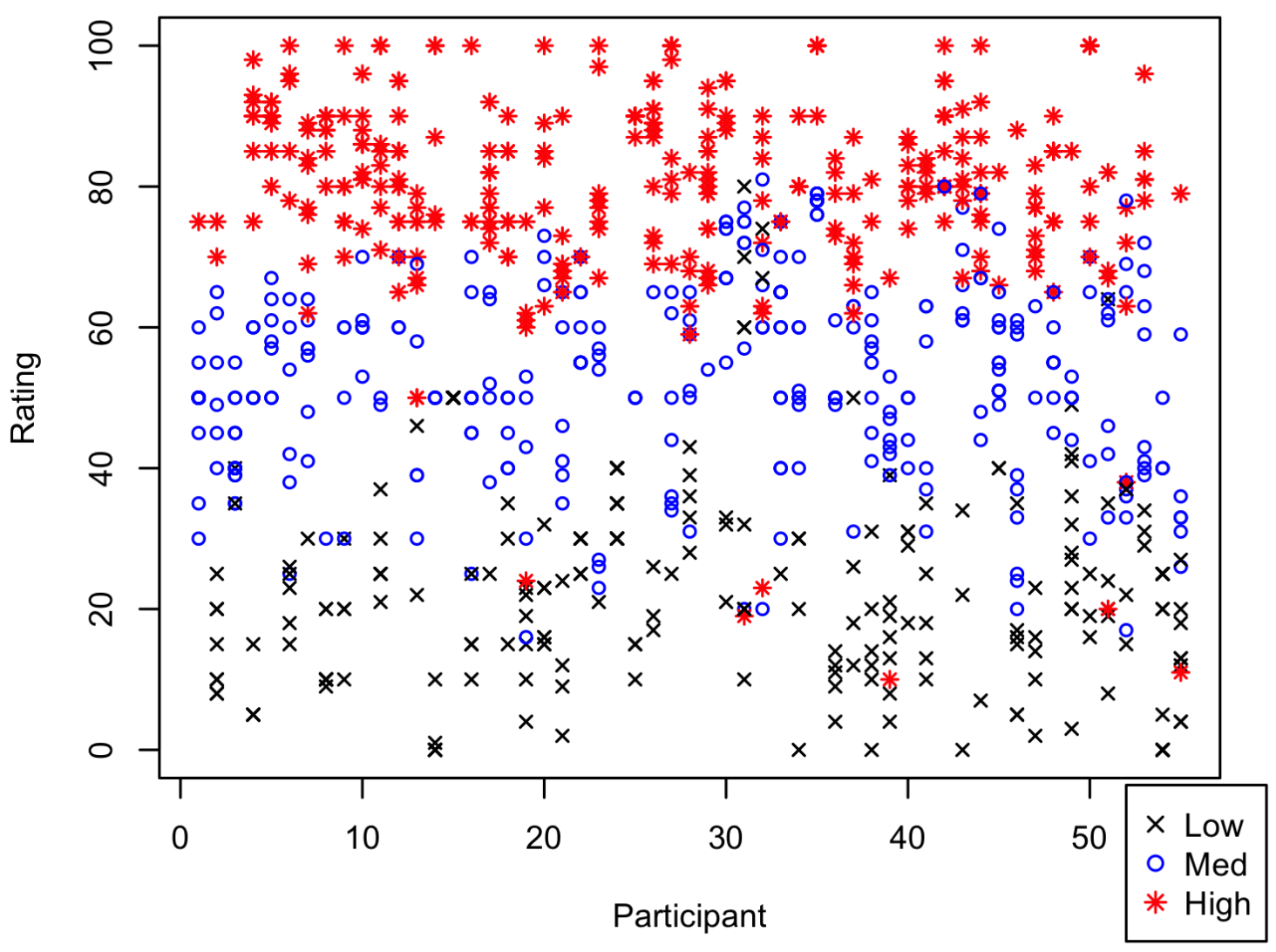

Figure 3. Overview of Survey Responses by Participant Number 
Even though participants could choose any RISK RATING between 0 and 100, the actual ranges reflected in the RISK RATING scores in Figure 3 varied widely across participants. For example, the ratings assigned by participants 20 and 21 were relatively dispersed across the 0 100 scale, while participants 1 and 24 restricted their responses to less than half of the available range. In addition, we observed that a couple of participants occasionally assigned RISK RATINGS that were inconsistent with their NIST RANK scores. For example, participant 19 rated several scenarios near a value of 22 on the RISK RATING scale, but while two of these scenarios were judged to have a NIST RANK of "low" risk, one of these scenarios was assigned a "high" NIST RANK. From these observations, we note that it is vital to include participantspecific terms in any modeling of the results in order to compensate for the differences in how individuals used the available response range.

Figure 4 shows the distribution of RISK RATING scores for each of the 16 scenarios using box plots. A key at the top of the figure indicates the levels of each of the factors (shown using checkmarks) that were associated with each of the scenarios. The horizontal black bar indicates the median rating for each scenario and the box represents the interquartile range. Scenarios are ordered along the X-axis according to the ranks of mean RISK RATING scores. Outliers, which occurred in scenarios 6,8 , and 11 , are depicted with circles. We can see that there is a significant difference in perceived risk between the least risky scenarios (including 15 and 7) and the most risky scenarios (including 6 and 11). Again, we do not remove any of the outlying judgments in our modeling. We note that if anything, removing these outliers would strengthen our conclusions by making the riskiest scenarios even more distinct. 


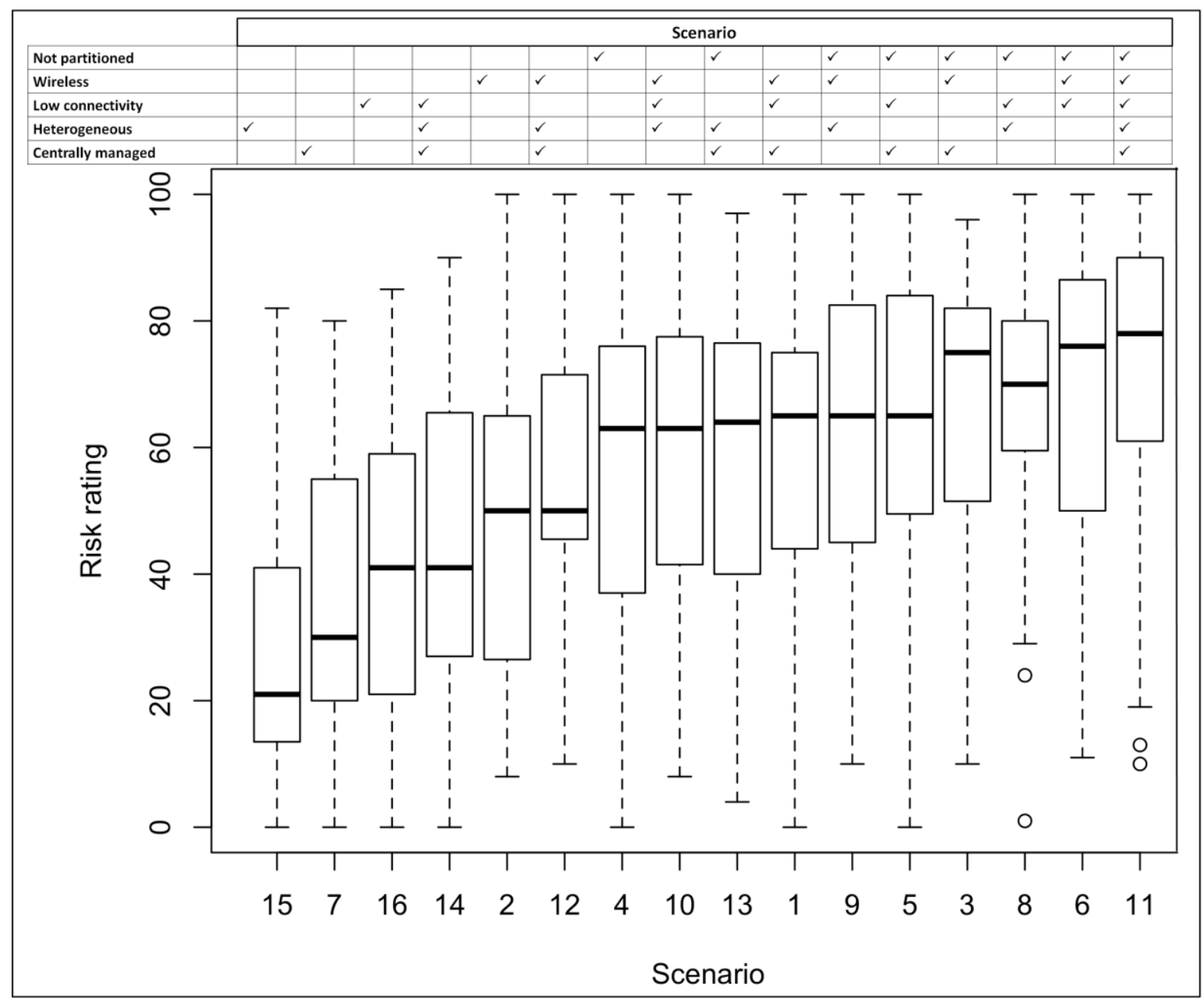

Figure 4. Boxplots of the Risk Rating Data by Scenario.

Figure 5 is a stacked bar plot depicting the frequencies of NIST RANK scores (low, medium, high) for each of the 16 scenarios. Scenarios are ordered along the X-axis as in Figure 4, and once again the key at the top of the figure indicates levels of factors associated with the scenarios. Examination of Figures 4 and 5 allows a comparison of the median RISK RATINGS with the frequency of NIST RANK data for each of the 16 scenarios. Participants seem to agree that the most risky scenarios were scenarios 6 and 11 while the least risky were scenarios 15 and 7. 


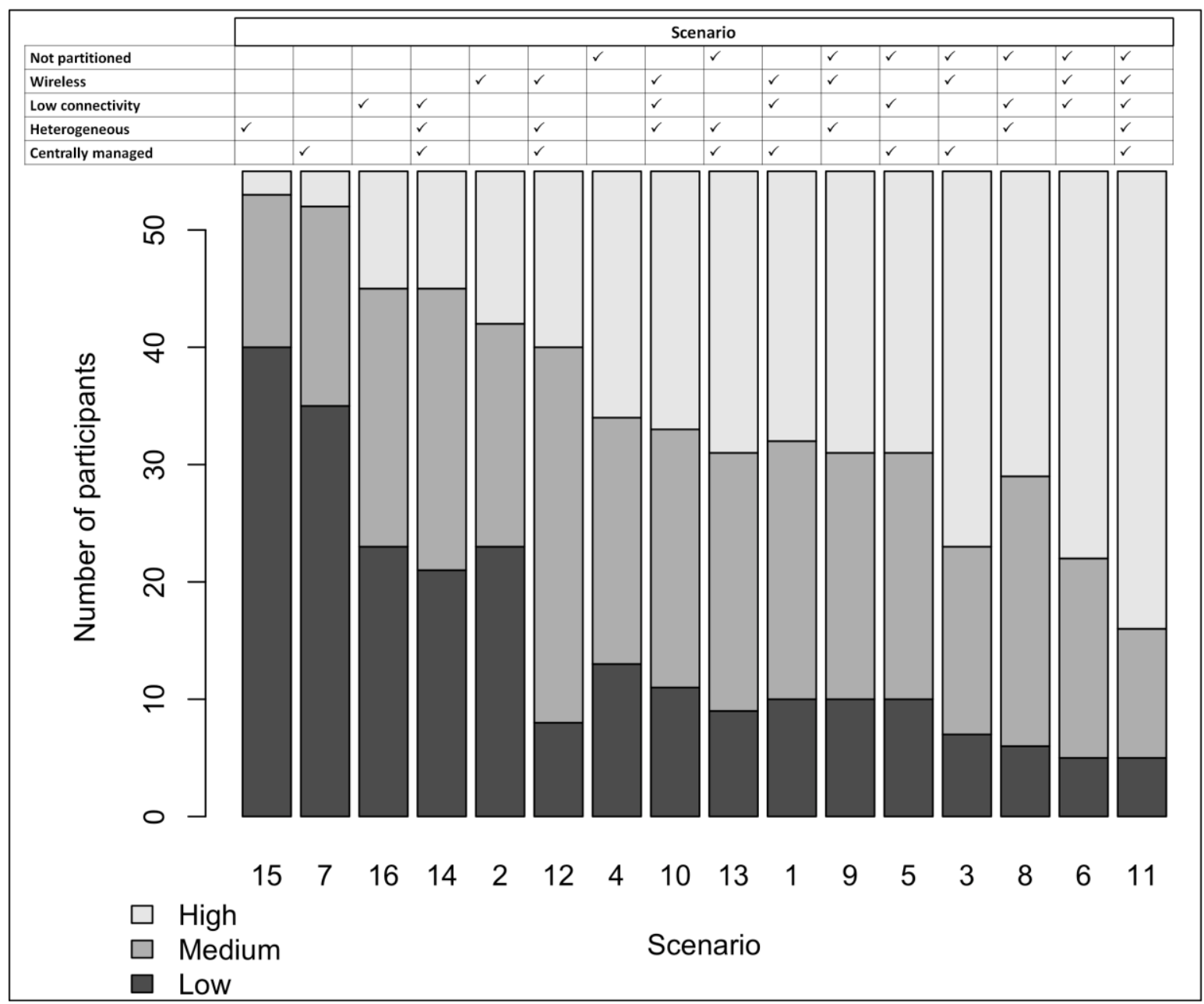

Figure 5. Bar plots (frequencies) of the Risk Ranking Data by Scenario.

\subsection{Statistical Modeling}

Major research questions for this study were whether any of the five network infrastructure factors (network partitioning, network diversity, wireless status, footprint, and connectivity) had a significant impact on the participants' judgment of network risk, and whether or not there were any two-way interactions among these factors (research question RQ1); and the extent to which a statistical model for the obtained data can account for group consensus on ratings and rankings (RQ2). We quantitatively addressed these questions through regression models of the risk ratings and rankings. 


\subsubsection{Analysis of Risk Ratings}

We first present the results of fitting a standard linear regression model to the RISK RATING data. In this model, the RISK RATING is the dependent variable and the five network infrastructure factors are predictor (independent) variables. Since individual participants varied greatly in the range of their assigned risk ratings (see Figure 3), we also included participant ID as a factor in the model. The ten two-way interactions of the five network infrastructure factors are also included as terms of the regression model. While it is common practice to standardize values of categorical predictor variables to aid interpretation of results in multiple regression analysis, it is not necessary to standardize binary independent factors (e.g., those that may be coded as 0's or 1's, such as the factors in our experimental design as described in Table 3), because their coefficients can already be directly interpreted (e.g., Gelman, 2008). Note that this regression model is equivalent to the random intercept mixed model, or the repeated measures ANOVA.

The fitted full model has four significant coefficients among the fixed effects (network factors and interactions) as well as a number of significant random intercept terms for the participants. Table 4 shows the estimated coefficients, standard errors, and p-values for the four significant effects (participant effects are excluded for conciseness, since they are not relevant to this research question).

Table 4. Table of regression coefficients for the full risk ratings model.

\begin{tabular}{|l|l|l|l|}
\hline Factor & $\begin{array}{l}\text { Estimated } \\
\text { Coefficient }\end{array}$ & $\begin{array}{l}\text { Std Error of } \\
\text { Coefficient }\end{array}$ & -value \\
\hline Network Partitioning & 26.08 & 2.88 & $<10^{-17}$ \\
\hline Wireless & 17.16 & 2.88 & $<10^{-8}$ \\
\hline Connectivity & 11.18 & 2.88 & 0.00012 \\
\hline Network Partitioning $\times$ Wireless & -10.92 & 2.58 & 0.00003 \\
\hline
\end{tabular}

As a standard linear regression model, the estimated coefficient for each factor indicates the expected change in mean RISK RATING for a network when changing the factor from level 0 to level 1 (see Table 2), controlling for all other factors. For example, the model predicts that changing a network from wired to wireless will increase perceived risk by 17.16 points. We see that Network Partitioning was extremely significant with the largest effect size. Changing a network from partitioned to not partitioned increased perceived risk substantially. Similarly, changing a network from wired to wireless also increased perceived risk by a large amount. Changing the connectivity of a network from high to low connectivity significantly increased 
perceived risk, but by less than partitioning or wireless status. The network footprint had a much smaller, non-significant impact. The only significant interaction was between network partitioning and wireless status. The effect of this interaction was negative, indicating that changing a network to not partitioned and wireless increased risk, but by less than would be expected from the sum of the two main effects.

Since a goal of this research is to discover a parsimonious model for estimating the risk of a network, we used a basic model selection technique to reduce the size of the model. Specifically, we used forward-backward stepwise regression with an AIC model comparison criteria to select a parsimonious model with only a subset of the network factors and interactions. The selected model still included the participant factor (random intercepts for each participant in the mixed model), and also included the five terms shown in Table 5. This reduced model is very similar to the full model's significant coefficients. Footprint appears as significant in this model, though with a very small effect size. The overall model was:

$$
\begin{aligned}
\text { Rating } & =(\text { participant-level intercept })+23.6 * \text { Network Partitioning }+17.4 * \text { Wireless Status } \\
& +8.2 * \text { Connectivity }-10.9 * \text { Network Partitioning*Wireless Status }
\end{aligned}
$$

Table 5. Table of regression coefficients for the selected factors of the risk ratings model.

\begin{tabular}{|l|l|l|l|}
\hline Factor & $\begin{array}{l}\text { Estimated } \\
\text { Coefficient }\end{array}$ & $\begin{array}{l}\text { Std Error of } \\
\text { Coefficient }\end{array}$ & $\boldsymbol{p}$-value \\
\hline Network Partitioning & 23.60 & 1.29 & $<10^{-34}$ \\
\hline Wireless Status & 17.40 & 1.29 & $<10^{-19}$ \\
\hline Connectivity & 8.22 & 1.29 & $<10^{-9}$ \\
\hline Footprint & 3.65 & 1.29 & 0.005 \\
\hline Network Partitioning $\times$ Wireless & -10.92 & 2.58 & 0.00003 \\
\hline
\end{tabular}

Both the full and reduced models include a participant intercept term that estimated the RISK RATING that a particular participant would judge a network when the five network factors are at their 0-level (partitioned, homogeneous, not wireless, distributed, high connectivity). Since all the models estimated positive or non-significant coefficients for the five factors, we expect this configuration to be judged the least risky. Figure 6 shows a histogram of the estimated values for this participant-level intercept. We can see that there is quite a lot of variability in where the participants place their ratings on the $[0,100]$ scale. This confirms what we found in exploratory analysis (Figure 3). 


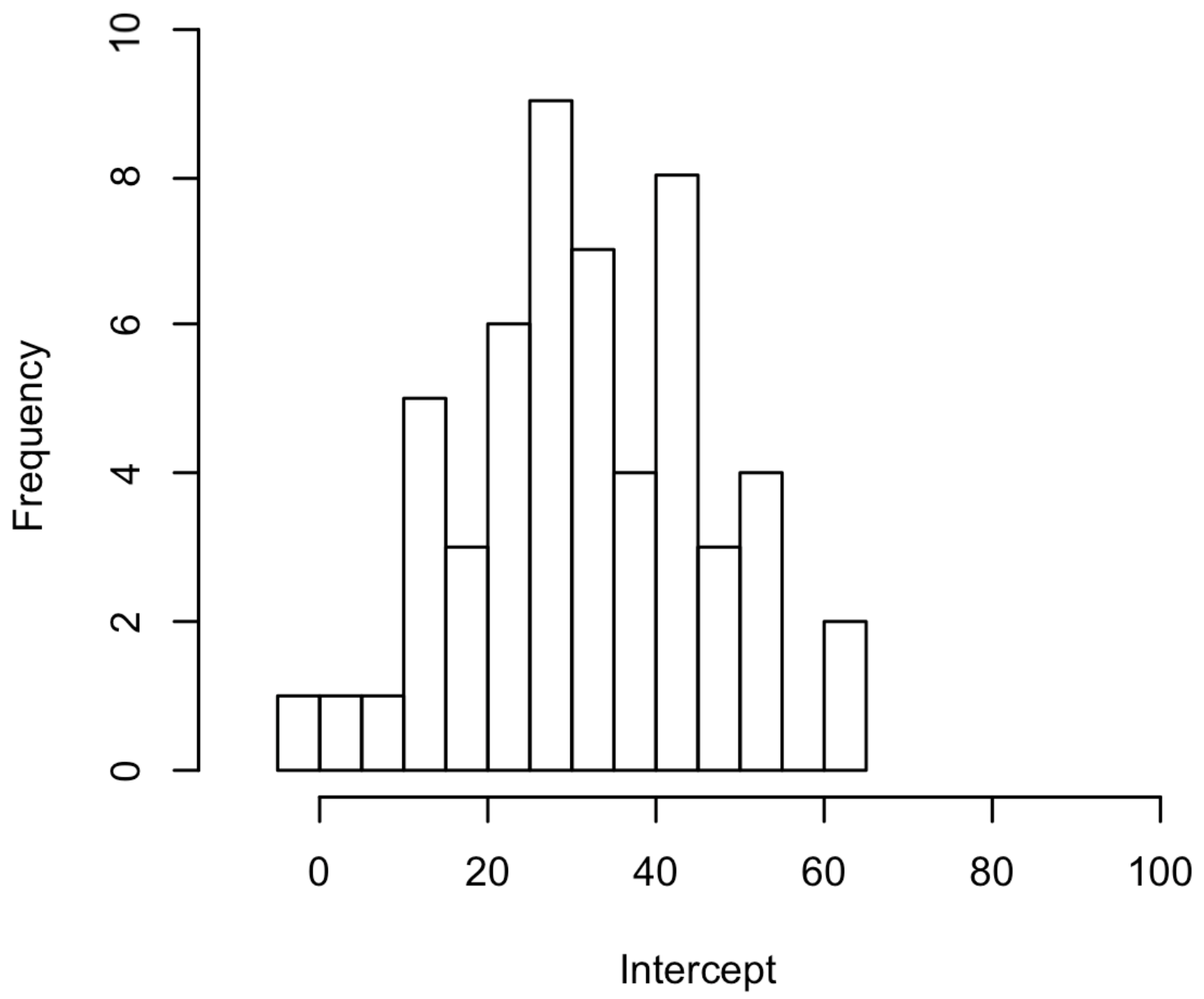

Figure 6. Histogram of participant-specific estimated intercept terms in linear model.

There is one participant whose estimated intercept is negative. This is due to the assumption in the linear model that the response variable is continuous and un-truncated. In reality, the risk ratings were limited to the range [0,100]. However, most participants did not use the full range. Standard model diagnostics revealed slight heteroscedasticity in the residuals due to small truncation effects from this limited range in the response variable. Due to the strong and consistent conclusions from this model and that of the NIST RANK data (Section 4.2.2, below), we do not judge this to be detrimental to our conclusions.

The linear model with random subject-specific intercepts accounts for differences in where the participants placed their ratings on the $(0,100)$ scale, but it does not account for any differences in the ranges of ratings used by the participants. We do not have sufficient responses from each participant to fit a random slope model that would estimate the effects of network factors for each individual participant. To address the differences in the width of the response 
ranges used, we can rescale the participants' responses in several ways. For instance, we can consider the ordering of the scenarios induced by each participant's ratings. That is, for each participant we give the scenario they considered least risky the value 1, and the scenario they considered most risky a 16 (averaging the values for tied scenarios). Alternatively, we could normalize each participant's responses to a particular range before analysis. Treating these new response scales as a linear response, we can fit a linear model as we did to the raw scores above. We find exactly the same pattern of significance and relative effect sizes as shown in Table 5. We conclude that the variability in the scaling the placement of responses across subjects is not detrimental to the extremely significant and robust patterns showing network partitioning, wireless status, and connectivity to be influential in the judgment of network risk.

Importantly, the adjusted $\mathrm{R}^{2}$ values for both the full and reduced RISK RATING models were approximately 0.47 , meaning that the models explain about $47 \%$ of the variance in risk ratings judgments. We hypothesize that the remaining, unexplained variability may come from uncontrolled factors including participant effects such as years and type of experience, interactions between participant and network effects, differences in the interpretation of the study tasks and questions, etc. Section 5 gives a more in-depth discussion of these limitations.

\subsubsection{Analysis of Risk Rankings}

Because the NIST RANK data were ordinal with three levels (low, medium, high), a different modeling approach was required. We used ordered logistic regression, also known as the ordered logit model or the proportional odds model. Again, we included a subject ID in the model to control for subject-level differences, the five main effects and the 10 two-way interactions. We again chose a reduced model using forward-backwards stepwise regression based on the AIC criteria. We also required that main effects be included in the model when they were involved in any included interactions.

The coefficients from the selected model are shown in Table 6. The table includes approximate $\mathrm{p}$-values computed using an asymptotic z-test, though these are likely biased due to a small sample size. Nevertheless, the results agree with those of the analysis of the RISK RATING model in the previous section, with the same significant main effects and the partitioning-wireless interaction, and with relative effect sizes that are comparable. Note that the estimated effect in these models describes the change in the log odds ratio. Positive values indicate an increase in the probability of a higher NIST RANK, while negative values indicate a decrease in the probability of a higher NIST RANK. The precise odds of each NIST RANK value (low, medium, high) can only be calculated with the subject-specific offset terms which we omit from table 6 for brevity. Since subjects differed in whether they centered their NIST RANK judgments on the high or low end of the scale, the changes in odds due to the network factors (given in table 6) are more general than specific values for individual participants. 
We can see from Table 6 that the risk rankings model shows similar effects to the risk ratings model. Although the model selection technique produced a slightly bigger model, including several marginally significant interaction effects not present in the RISK RATING models, the highly significant effects are again those of network partitioning, wireless status, connectivity and the partitioning $X$ wireless interaction. Refer to Figure 5 for the frequency of low, medium and high NIST RANKs across all 16 scenarios. Though additional coefficients appear as marginally significant with small effect sizes, due to the bias in the $p$-value calculation and the small effect sizes, we do not think these should be interpreted as meaningful without further study.

Table 6. Table of regression coefficients for the selected factors of the risk rankings model.

\begin{tabular}{|l|l|l|l|}
\hline Factor & Estimated Effect & $\begin{array}{l}\text { Std Error of } \\
\text { Effect }\end{array}$ & $\begin{array}{l}\text { Approx. } \text { - } \\
\text { value }\end{array}$ \\
\hline Network Partitioning & 2.85 & 0.28 & $<10^{-25}$ \\
\hline Wireless Status & 1.94 & 0.22 & $<10^{-19}$ \\
\hline Connectivity & 1.57 & 0.26 & $<10^{-9}$ \\
\hline Footprint & 0.40 & 0.25 & 0.115 \\
\hline Network Partitioning x Wireless Status & -1.12 & 0.3 & 0.00016 \\
\hline Network Diversity & -0.19 & 0.21 & 0.37 \\
\hline Network Partitioning x Connectivity & -0.63 & 0.29 & 0.032 \\
\hline Network Diversity x Footprint & 0.64 & 0.29 & 0.03 \\
\hline Footprint x Connectivity & -0.63 & 0.29 & 0.03 \\
\hline
\end{tabular}

\section{Discussion}

This study experimentally manipulated five network topology factors to determine the impacts of these factors and their interactions on judgments of network risk. Both research questions were interrelated: RQ1 assessed the relationship between network topology factors and network risk judgments and RQ2 evaluated group consensus on ratings and rankings with respect to model fit. In subsections 5.1 and 5.2 we discuss the findings relating to these research questions in turn; we conclude by discussing implications of our findings for research and practice (subsection 5.3) and limitations of the research (subsection 5.4).

\subsection{Judgments of Network Risk (RQ1)}

First we summarize the results and then we attempt to explain or rationalize these findings from an operational perspective.

\subsubsection{Summary of Results}


In our research effort to address RQ1, we've found that three network factors (partitioning, wireless status, and connectivity), and one interaction (partitioning $X$ wireless) exhibited extremely significant and consistent relationships with network risk judgments. The remaining two factors and 9 interactions were not significant in models of perceived risk. Therefore, we conclude that some network topological factors are very important to network risk judgments, and that the interactions cannot be ignored. To summarize the significant results found:

- $\quad$ Network partitioning. Compared to a partitioned network, a non-partitioned network has a much higher predicted perceived risk; and this difference is greater for this factor than for any other factor studied.

- Wireless status. A wireless network has a somewhat higher predicted perceived risk than a wired network.

- Connectivity. A network that is not available, i.e., with low connectivity, has a slightly higher predicted perceived risk, but the difference is smaller than that resulting from changing network partitioning or wireless.

- Network partitioning X wireless status. The effect was negative and relatively small, meaning that while the perceived risk of a network is increased when it is changed to be both wireless and not partitioned, the increase is less than would be expected from the two main effects alone. Since both main effects were larger than the interaction effect, a non-partitioned, wireless network is still the most risky combination of those two factors.

\subsubsection{Operational Perspective}

These findings can be further explained with respect to operations. Prior research indicated that non-partitioned networks have more potential to lose the most data in an adversarial attack (e.g., Scarfone et al., 2011). When one non-partitioned network exists that contains all machines and resources that are configured and managed the same way, an adversary can access all machines and their resources after compromising only a single machine on that network. The risk is inflated when wireless connections between machines exist but this inflation is lower than expected when wireless and not-partitioned networks were evaluated. Partitioning the network creates smaller subgroups of machines and resources. An adversary who gains entry into a subgroup can only access resources and data within that subgroup. Disadvantages to partitioning networks are that it requires more resources and there is a risk of negatively impacting worker productivity as access to network data becomes more restricted. If needed resources go down in one subgroup, those group members halt productivity until resources are restored. We cannot say whether or not our participants were conscious of the tradeoff between partitioning and productivity, nor whether that awareness would have impacted their risk assessments. The concept of connectivity dovetails with this discussion. 
According to NIST FIPS Publication 199 (pp.2), availability is defined as "Ensuring timely and reliable access to and use of information..." [44 U.S.C., SEC. 3542]. "A loss of availability is the disruption of access to or use of information or an information system." Connectivity to the internet is one way that availability is represented. When a network architects design a network, maximizing availability for authorized users while minimizing availability for adversaries might be a typical goal. In live operations, authorized users typically have different levels of permissions that are difficult to manage, so reducing network partitioning and aggregating resources on a small number of networks is one way to reduce the management burden. Typically as a consequence, the number of connections to the internet is also reduced, which reduces the number of avenues available to a malicious actor from the internet. However, aggregating resources on a small number of networks means that authorized users may have access to each other's resources without an explicit need; this can increase the likelihood that a malicious insider can access data and resources. Therefore, increasing the availability and connections to the internet may increase chances of access by outside malicious actors, but reducing the availability and connections to the internet may increase chances of access by malicious insiders.

\subsection{Consistency of Network RiskJudgments (RQ2)}

First, we discuss the main findings regarding our statistical modeling approach to assessing the consistency of network risk judgments and then we address considerations relating to the quality of the fit and sources of variability for these models.

\subsubsection{Summary of Results}

RQ2 concerned the consistency of participant judgments. We addressed this question through exploratory analysis and by examining the quality of our models' fit to the data. Consistency of judgments was assessed at the scenario level (within-subject and across subjects) in Figures 4 and 5. These figures show substantial variability in the range of scenario ratings and rankings used by participants. The size of the interquartile range of box plot box plots in Figure 4 reflects the variability of RISK RATINGs of overall risk judgments by scenario 8, 12 and 15 . We also can infer agreement by looking at relative frequencies of NIST RANKINGs (Figure 5); most people agreed that scenario 11 was high risk while scenario 15 was low risk.

\subsubsection{Sources of Variability in Network Risk Judgments}

By assessing model fit, we can determine how much of the obtained variability can be attributed to the experimentally manipulated network factors. Our models explained $\sim 47 \%$ of the variance (the best fitting models had an $\mathrm{R}^{2}$ of 0.47 ) in the risk judgments, yet we still found highly significant and robust main effects. Half of the variance was left unexplained in this statistical model. This $\mathrm{R}^{2}$ value compares very favorably to results from research in the social sciences, 
where correlations of this magnitude are characterized as indicating a very large effect size (Cohen, 1988; Rosenthal, 1996). Thus, our linear equation is a computational model of network risk judgments that can serve as a baseline model, which may be compared to other models or metrics for information system risk.

The large effect size notwithstanding, here we offer some hypotheses that might account for the remaining unexplained variance. First, the diagnostics revealed slight heteroscedasticity in the residuals due to truncation of the rating scale. This may have reduced the overall model fit but did not seem to impact the robustness of the significant main effects and the interactions. Second, a review of Figure 3 indicates that respondents use the response scales differently which is not unusual from a psychometric perspective. Figure 6 shows the subject-level effect that resulted when we modeled the intercept term which describes where participants place the least risky scenarios on the response scale. Psychometric response scale research has documented how different response scale formats yield response biases (Cowley \& Youngblood, 2009; Schaeffer \& Presser, 2003; Schaeffer \& Barker, 1995; Schwarz, Knauper, Hippler, Noelle-Neumann \& Clark, 1991) so reformatting response scales does not necessarily correct these biases. Tradeoffs exist across response scale formats with respect to measurement quality, ease of use, consistency of ratings, and validity (Krosnick \& Fabrigar, 1997; Andrews, 1984). Another potential source of variability from the NIST RANKING categorical scale is that it is unclear whether a jump between a ranking of low to medium is equal in importance to a jump between medium to high; a problem common to ordinal scales (Bradburn, Sudman \& Blair, 1980).

Another contributing factor to unexplained variance is judgment biases based on previous participant experiences with network topologies. We originally used text-based factor scenarios that described different factor levels but found that people were basing judgments on descriptive terminology for different factor levels so we assumed that by moving to pictorial representations of scenarios with no text, the unexplained variance would be reduced. Yet, upon the review of simplified network topologies, we asked participants questions that most likely required a story behind the topology to answer the question. Everyone may have used a different story.

In sum for RQ2, our exploratory and statistical analyses indicated that the particular values of RISK RATING and NIST RANK assigned to each scenario depended heavily on the participant. That is, though several network factors reliably and strongly changed the perceived risk across participants, the actual number or rank was subject-specific. We saw this variability in the exploratory analysis, and also in the significant subject-specific terms in all of our models. The fact that we found extremely significant and large effects of network features even in the face of this variability speaks to the robustness of these effects. This study provides evidence that these characteristics are important to consider in any model of network risk. At the same time, the variability among experts demonstrated here suggests that great care should be put into standardizing and clarifying the expectations for any subjective scales used in network risk assessment. 


\subsection{Implications for Research and Practice}

This section ties our study results back to the introduced problem and related research. As discussed at the beginning of this manuscript, human judgment is impacted by other elements of cognition (e.g., risk attitudes, cognitive biases, fatigue) and of the environment (e.g., organizational policies, culture, rate of adversarial activity). There is also a temporal component to network risk judgment. We scoped this effort to study only the relationship between the perception of network topological factors and risk judgment in order to lay the foundation of future, systematic, empirical investigation of network risk judgment. We caution the reader not to exaggerate the implications of our findings to the improvement of risk metrics, because one study is hardly enough evidence to explain the relationship between network risk perception and risk judgment. But we aim to start discussions on how to best improve network risk metrics. The results of our study conjure up several implications, but for brevity we focus on one implication for the academic community and one for the operational community.

For the academic community, we revisit the concept of group agreement that serves as a proxy for ground truth. What is group agreement for socially constructed, multidimensional phenomenon and how do we best measure this for operational purposes? First, we cannot ignore the fallacy of the circular argument ( $\mathrm{A}$ is true because of B and B is true because of $\mathrm{A}$ ) that group consensus is a proxy for ground truth. For our problem, the circular argument would be phrased as, "ground truth is group consensus because group consensus is said to be ground truth for the 'true network risk level'". At best, network risk judgments that align with group consensus provide some degree of confidence that these judgments are relatively more accurate than judgments that do not align with group consensus; but group consensus may just be the outcome of a sample's heuristic-based decision making. We did find agreement that certain factors are important to network risk, but participants did not exhibit consistent ranges in their risk ratings for each factor level, nor did they always agree on whether a factor level should be ranked as low, medium or high risk.

Second, it is not always clear what the most rigorous approach is to the measurement of socially constructed phenomenon; it depends on the use-case of the metric. From psychometric theory, ipsative and normative metrics are two types of use-case specific metrics. Ipsative metrics, also referred to as forced-choice metrics, are often used to measure the relative value of phenomena (for instance, perceived risk across networks) within a unit of observation (for instance, a single cyber expert) (Chan, 2003; Jackson and Alvin, 1980). Ipsative metrics are often used to measure subjective interpretation of psychological constructs (van Eijnatten, van der Ark, \& Holloway, 2014). In contrast, normative metrics aim to obtain absolute measures of a phenomenon (for instance perceived risk) that are comparable across people. Whether the metric is ipsative or normative can impact its reliability and validity for answering particular questions (van Eijnatten, van der Ark, \& Holloway, 2014). Ipsative measures improve the validity of metrics that have significant response bias by human judgers (Hicks, 1970) but the reliability 
consequently suffers (Saville \& Wilson, 1991; Bartram, 1996). Ipsative metrics also have been known to correct for acquiescence responding, faking or impression management, and social desirability bias (Cunningham, Cunningham \& Green, 1977; McLean \& Chissom, 1986; Gurwitz, 1987). We observed several potential response biases in our data that might be addressed using ipsative metrics instead of normative metrics. Ipsative metrics might correct some of these issues, but it is not yet clear whether they would yield valid or useful measures in the context of computed risk models. Future research could examine existing models of computed risk in the context of psychometric theory. Additional experimental work might validate and quantify the benefits and tradeoffs of these various metric design decisions in the network risk context.

The implications to the operational community involve the computational model we derived. Some of the computational models on which commercially-available network risk metrics are based include a combination of objective (e.g., "number of assets damaged") and subjective (e.g., "moderate damage or loss") components. Reducing the number of subjective components from a computed model may severely compromise the validity of the metric because risk is socially constructed. Some have argued that current practice relies too much on subjective judgments and lacks quantitative rigor (e.g., Longstaff et al., 2000; Pamula et al., 2006); but quantitative rigor of subjective phenomenon is, in part, contingent upon the consistency judgments across the population given a particular cue pattern of perceived factors in a network. Quantitative rigor is also contingent upon explained vs. unexplained variance in a model. In this study, we formulated a computed risk model (i.e., the linear equation of our regression model) based exclusively on subjective human judgments of network risk. While we found three factors with robust significant main effects, the model explained only $\sim 47 \%$ of the variance in subjective judgments; the more variance explained, the better the model-this is very good by social science standards, but what are the implications for operational use? The direct implication is that risk judgments using this computational model may yield unacceptable error rates. Given the need for rigorous risk metrics to evaluate mission-critical information systems, is this an acceptable level of explained variance for computed risk models? To our knowledge, no similar benchmark evaluations of computed risk models have been conducted. Continued research is needed to understand how we can improve these models and respective metrics.

\subsection{Limitations}

Four limitations are important for researchers interested in building upon this work. First, we did not offer participants a definition of network risk to standardize the RISK RATINGS because we wanted them to provide ratings based on their own. However, we did not document what that personal definition was. After participants used their own definition for the RISK RATINGS, we then tried to standardize their NIST RANKING responses by provided them NIST guidance. As participants worked through subsequent scenarios, we failed to explicitly direct them to use their 
own definition of risk when providing the RISK RATING. Therefore, after the first RISK RATING and NIST RANKINGS were provided, we may have actually documented ratings and rankings that converge on the NIST guidance rather than obtaining ratings based on their own definition. Therefore, we recommend collecting participants personal risk definition prior to providing any ratings and rankings and then being explicit about which definition participants should use for each type of response they provide.

A second limitation involves an ambiguity in the risk ranking question. We used the NIST guidance on risk ranking level (low/medium/high) that asks participants to assess network risk based only on impact, rather than likelihood and impact. Specifically, the rankings were differentiated based on the expected monetary and reputation costs of a successful network intrusion and whether this would cause injury or death to humans. However, since our experimental materials provided only network topology, without additional context about the organization being assessed, the scenarios lacked information necessary to assess monetary, reputation, personnel, or other impacts. Furthermore, we cannot be certain if participants read and based their responses on the NIST risk level guidance that was provided.

Third, we acknowledge that metrics based on network topology provide only a partial view of network risk (Zhang, Ou, Singhal, \& Homer, 2011; Idika \& Bhargava, 2012; Wang, Jajodia, Singhal, Cheng \& Noel, 2014). We used a factor scenario method (Rossi, 1951; Rossi, 1979) from the social sciences that does not explicitly tell the participants what factors are present and when each factor is manipulated across each scenarios. By telling a participant what factor manipulations are changing from one scenario to the next, we unnaturally can cue participants to change their ratings and ranking accordingly when this would have not happened otherwise. Also, we cannot guarantee that participants perceived the manipulations made across the network topologies even though our beta testing revealed that the manipulations were perceivable. Failure to perceive may be due to any number of cognitive mechanisms (perceptual biases, fatigue, memory lapse, etc.). Hence a risk judgment cannot always be confidently related to changes in the manipulated network factors.

Finally, if researchers are interested in generating a model that explains more variance, study modifications might enable that. We suspect that there are differences in the impact of each of the factors between participants. That is, participants scale their responses differently, and may disagree on the magnitude of the influence of each factor. However, to formally assess the amount of disagreement about each factor across the population, we would need each individual to rate more networks. This additional data would allow the fitting of models with subjectspecific factor terms. These models could reveal the extent of systematic agreement or disagreement between experts about the impact of specific network factors on perceived risk. This could be important for designing computed risk models. If we want to create a model that describes consensus across experts, we may wish to favor factors with high agreement. 


\section{Acknowledgments}

Copyright 2014 Carnegie Mellon University. This material is based upon work funded and supported by the Department of Defense under Contract No. FA8721-05-C-0003 with Carnegie Mellon University for the operation of the Software Engineering Institute, a federally funded research and development center.

NO WARRANTY. THIS CARNEGIE MELLON UNIVERSITY AND SOFTWARE ENGINEERING INSTITUTE MATERIAL IS FURNISHED ON AN “AS-IS" BASIS. CARNEGIE MELLON UNIVERSITY MAKES NO WARRANTIES OF ANY KIND, EITHER EXPRESSED OR IMPLIED, AS TO ANY MATTER INCLUDING, BUT NOT LIMITED TO, WARRANTY OF FITNESS FOR PURPOSE OR MERCHANTABILITY, EXCLUSIVITY, OR RESULTS OBTAINED FROM USE OF THE MATERIAL. CARNEGIE MELLON UNIVERSITY DOES NOT MAKE ANY WARRANTY OF ANY KIND WITH RESPECT TO FREEDOM FROM PATENT, TRADEMARK, OR COPYRIGHT INFRINGEMENT.

This material has been approved for public release and unlimited distribution. Carnegie Mellon ${ }^{\circledR}$ is registered in the U.S. Patent and Trademark Office by Carnegie Mellon University. DM0001515 


\section{References}

Aagedal, J. O., Den Braber, F., Dimitrakos, T., Gran, B. A., Raptis, D., \& Stolen, K. (2002). Model-based risk assessment to improve enterprise security. In Proceedings of the 6th International Enterprise Distributed Object Computing Conference. 51-62.

Ahmed, M. S., Al-Shaer, E., and Khan, L. 2008. A Novel Quantitative Approach For Measuring Network Security. INFOCOM 2008. The 27th Conference on Computer Communications. IEEE, 76-80. http://ieeexplore.ieee.org/xpl/login.jsp?tp=\&arnumber $=4509855 \&$ url $=\mathrm{http} \% 3 \mathrm{~A} \% 2 \mathrm{~F} \% 2 \mathrm{Fiee}$ xplore.ieee.org\%2Fxpls\%2Fabs_all.jsp\%3Farnumber\%3D4509855

Alberts, C., and Dorofee, A. 2001. Operationally Critical Threat, Asset, and Vulnerability Evaluation (OCTAVESM) Method Implementation Guide, v2.0. Software Engineering Institute, Carnegie Mellon University. http://www.cert.org/octave/octavemethod.html.

Andrews, F. M. (1984). Construct validity and error components of survey measures: A structural modeling approach. Public opinion quarterly, 48(2), 409-442.

Aven, T. (2003). Foundations of Risk Analysis: A Knowledge and Decision-Oriented Perspective. John Wiley \& Sons Ltd. (ISBN 0-471-49548-4).

Ajzen, I. (1991). The theory of planned behavior. Organizational behavior and human decision processes, 50(2), 179-211.

Ajzen, I., \& Fishbein, M. (1977). Attitude-behavior relations: A theoretical analysis and review of empirical research. Psychological bulletin, 84(5), 888.

Baran, P. (1964). On Distributed Communications Networks. IEEE Transactions on Communications Systems, 12 (1), pp. 1-9. 10.1109/TCOM.1964.1088883. http://ieeexplore.ieee.org/xpl/login.jsp?tp=\&arnumber=1088883\&url=http\%3A\%2F\%2Fieee xplore.ieee.org\%2Fxpls\%2Fabs_all.jsp\%3Farnumber\%3D1088883 (accessed April 15, 2014).

Bartram, D. (1996). The relationship between ipsatized and normative measures of personality. Journal of Occupational and Organizational Psychology, 69(1), 25-39. 
Bauer, R. A. (1960). Consumer Behavior as Risk Taking, 389 - 398. In R. E. Karp (Ed.), Issues in Marketing. MSS Information Corporation. (ISBN 0-8422-5165-0).

Biernacki, P. \& Waldorf, D. (1981). Snowball Sampling: Problems and Techniques of Chain Referral Sampling. Sociological Methods \& Research 10(2), 141-163.

Bleich, D. (1978). Subjective criticism. Baltimore: Johns Hopkins University Press.

Boring, E. G. (1930). A new ambiguous figure. The American Journal of Psychology.

Bradburn, N. M., Sudman, S., \& Blair, E. (1980). Improving interview method and questionnaire design: Response effects to threatening questions in survey research. San Francisco, CA: Jossey-Bass.

Breier, J., \& Hudec, L. (2011, June). Risk analysis supported by information security metrics. In Proceedings of the 12th International Conference on Computer Systems and Technologies (pp. 393-398). ACM.

Buchanan, B. G., Davis, R., \& Feigenbaum, E. A. (2006). Expert systems: a perspective from computer science. The Cambridge handbook of expertise and expert performance, 87-103.

Chan, W. (2003). Analyzing ipsative data in psychological research. Behaviormetrika, 30(1), 99121.

Clemente, R., Bartoli, M., Bossi, M. C., D'orazio, G., \& Cosmo, G. (2005, October). (2005). Risk management in availability SLA. In 5th International Workshop on Design of Reliable Communication Networks (DRCN 2005). Proceedings. IEEE, pp. 8-pp.

Cohen, J. (1988). Statistical power analysis for the behavioral sciences (2nd ed.). Hillsdale, NJ: Erlbaum

Cowan, N. (1997). Attention and memory. Oxford University Press.

Cowley, J., Greitzer, F. L., and Woods, B. (2015). Factors Influencing Network Risk Perception: A Conceptual Inquiry and Exploratory Analysis. Security Informatics, 4(1). doi:10.1186/s13388-015-0016-x 
Cowley, J. A., \& Youngblood, H. (2009, October). Subjective response differences between visual analogue, ordinal and hybrid response scales. In Proceedings of the Human Factors and Ergonomics Society Annual Meeting (Vol. 53, No. 25, pp. 1883-1887). Sage Publications.

Crespo, A. H., del bosque, R., \& de los Salmones Sanchez, G. (2009). The Influence of Perceived Risk on Internet Shopping Behavior: A Multidimensional Perspective. Journal of Risk Research 12(2), 259-277.

Cronbach, L. J. \& Meehl, P. E.(1955). Construct Validity in Psychological Tests. Psychological Bulletin 52 (4), 281-302.

Cunningham, W. H., Cunningham, I. C., \& Green, R. T. (1977). The ipsative process to reduce response set bias. Public Opinion Quarterly, 41(3), 379-384.

Dallenbach, K. M. (1951). A puzzle-picture with a new principle of concealment. The American journal of psychology.

Diener, E. (2000). Subjective well-being: The science of happiness and a proposal for a national index. American psychologist, 55(1), 34.

Dixon, P., Bortolussi, M., Twilley, L. C., \& Leung, A. (1993). Literary processing and interpretation: Towards empirical foundations. Poetics, 22(1), 5-33.

Dowling, G. R. (1986). Perceived Risk: The Concept and Its Measurement. Psychology \& Marketing, 3, 193-210.

Eagly, A. H., \& Chaiken, S. (1993). The psychology of attitudes. Harcourt Brace Jovanovich College Publishers.

Edelman, S., \& Weinshall, D. (1991). A self-organizing multiple-view representation of 3D objects. Biological Cybernetics, 64(3), 209-219.

Elky, S. 2006. An Introduction to Information System Risk Management. SANS Institute: InfoSec Reading Room. http://www.sans.org/reading-room/whitepapers/auditing/introductioninformation-system-risk-management-1204. 
Ericsson, K. A., \& Lehmann, A. C. (1996). Expert and exceptional performance: Evidence of maximal adaptation to task constraints. Annual review of psychology, 47(1), 273-305.

Fischhoff, B. (2009). Risk Perception and Communication. In R. Detels, R. Beaglehole, M. A. Lansang, \& M. Gulliford (Eds.), Oxford Textbook of Public Health, Fifth Edition. Oxford University Press: Sage, 940 - 952.

Fischhoff, B., Detels, R., Beaglehole, R., Lansing, M. A., \& Gulliford, M. (2009). Risk perception and communication. Oxford textbook of public health, Volume 2: the methods of public health, (Ed. 5), 940-953.

Gelman, A. (2008). Scaling regression inputs by dividing by two standard deviations. Statistics in Medicine, 27, 2865-2873.

Gemünden, H. G. (1985). Perceived Risk and Information Search: A Systematic Meta-Analysis of the Empirical Evidence. International Journal of Research in Marketing 2(2),79-100.

Gandhi, R. A., \& Lee, S. W. (2011). Discovering Multidimensional Correlations among Regulatory Requirements to Understand Risk. ACM Transactions on Software Engineering and Methodology (TOSEM), 20(4), 16.

Gauthier, I., \& Tarr, M. J. (1997). Orientation priming of novel shapes in the context of viewpoint-dependent recognition. Perception, 26, 51-73.

Gilovich, T., Griffin, D., \& Kahneman, D. (Eds.). (2002). Heuristics and biases: The psychology of intuitive judgment. Cambridge University Press.

Guilford, J. P. (1952). When not to factor analyze. Psychological Bulletin, 49(1), 26.

Gurwitz, P. M. (1987). Ipsative rescaling: An answer to the response set problem in segmentation analysis. Journal of Advertising Research.

Haimes, Y. Y. (2009). On the Complex Definition of Risk: A Systems-Based Approach. Risk Analysis 29(12), 1647-1654. 
Hart, S. G., \& Staveland, L. E. (1988). Development of NASA-TLX (Task Load Index): Results of empirical and theoretical research. Advances in psychology, 52, 139-183.

Hicks, L. E. (1970). Some properties of ipsative, normative, and forced-choice normative measures. Psychological bulletin, 74(3), 167.

Hirsch, E. D. (1978). The aims of interpretation. University of Chicago Press.

Idika, N., \& Bhargava, B. (2012). Extending attack graph-based security metrics and aggregating their application. IEEE Transactions on Dependable and Secure Computing, 9:75-85.

Ingene, Charles A. \& Hughes M. A.(1985). Risk Management by Consumers. In E.C. Hirschman (Ed.), Research in Consumer Behavior, Vol. 1. Emerald Group Publishing Limited, 103-158.

Jackson, D. J., \& Alwin, D. F. (1980). The factor analysis of ipsative measures. Sociological Methods \& Research, 9(2), 218-238.

Jojodia, S., Noel, S., and O’Berry, B. (2005). Topological analysis of network attack vulnerability. In Kumar, V, Srivastava, J, and Lazarevic, A. (Eds.), Managing Cyber Threats: Issues, Approaches, and Challenges. Springer, ISBN 978-0-387-24230-9. pp. 247-266.

Karygiannis, T., and Owens, L. (2002). Wireless Network Security. NIST: Special Publication 800-48. http://m.tech.uh.edu/faculty/conklin/IS7033Web/7033/Week9/NIST_SP_800-48.pdf (accessed April 15, 2014).

Krosnick, J. A., \& Berent, M. K. (1993). Comparisons of party identification and policy preferences: The impact of survey question format. American Journal of Political Science, 941-964.

Krosnick, J. A., \& Fabrigar, L. R. (1997). Designing rating scales for effective measurement in surveys. Survey measurement and process quality, 141-164.

Kukla, A. (2000). Social constructivism and the philosophy of science. Psychology Press.

Longstaff, T.A., Chittister, C., Pethia, R. \& Haimes, Y.Y. (2000). Are we forgetting the risks of information technology? IEEE Computer, 33(12), pp. 43-51. 
McCrae, R. R., \& Costa, P. T. (1987). Validation of the five-factor model of personality across instruments and observers. Journal of personality and social psychology, 52(1), 81.

McLean, J. E., \& Chissom, B. S. (1986). Multivariate Analysis of Ipsative Data: Problems and Solutions.

Mell, P., Scarfone, K., and Romanosky, S. (2007). CVSS: A Complete Guide to the Common Vulnerability Scoring System Version 2.0. NIST Publication. http:/www.first.org/cvss/cvssguide.pdf

Miller, E. K. (1999). Neurobiology: Straight from the top. Nature, 401(6754), 650-651.

Montgomery, D. C. (2009). Design and analysis of experiments (Vol. 8). New York: John Wiley \& Sons.

National Institute of Standards and Technology (NIST). (2008). Performance Measurement Guide for Information Security. (NIST Special Publication 800-55, Revision 14) July 2008. http://csrc.nist.gov/publications/nistpubs/800-55-Rev1/SP800-55-rev1.pdf (accessed May 6, 2014).

National Institute of Standards and Technology (NIST). (1995). NIST Special Publication 80012: An Introduction to Computer Security - The NIST Handbook, Ch 12 (Security and Planning in the Computer System Life Cycle). Page 71.

National Institute of Standards and Technology (NIST). (2011). Managing Information Security Risk (NIST Special Publication 800-39). http://csrc.nist.gov/publications/nistpubs/80039/SP800-39-final.pdf

National Institute of Standards and Technology (NIST). (2012). Guide for Conducting Risk Assessments (NIST Special Publication 800-30 Revision 1). http://csrc.nist.gov/publications/nistpubs/800-30-rev1/sp800_30_r1.pdf (accessed April 15, 2014).

National Institute of Standards and Technology (NIST). (2013). Security and Privacy Controls for Federal Information Systems and Organizations. (NIST Special Publication 800-53, Revision 4) April 2013. http://nvlpubs.nist.gov/nistpubs/SpecialPublications/NIST.SP.80053r4.pdf (accessed April 15, 2014). 
Pamula, J., Jammann, P, Jajodia, S., \& Swarup,V. (2006). A weakest-adversary security metric for network configuration security analysis. 2nd Quality of Protection Workshop ( $Q o P^{\prime}$ (06): Security Measurements and Metrics, October 30, 2006, Alexandria, Virginia.

Peterson, M. A. (1999). What's in a stage name? Comment on Vecera and O'Reilly (1998).

Pfleeger, C. P. \& Pfleeger, S. L. (2003). Security in Computing 3rd Ed. Prentice-Hall.

Phillips, C., and Swiler, S.P. (1998). A graph-based system for network-vulnerability analysis. Proceedings of the 1998 workshop on new security paradigms (NSPW '98). New York, NY: ACM, 71-79.

Pollatsek, A. \& Tversky, A. (1970). A Theory of Risk. Journal of Mathematical Psychology, 7 , 540-553.

Poulton, E. C. (1989). Bias in Quantifying Judgments. Psychology Press.

Ragland, M. E. (1978). A New Kind of Humanism in the Literature Classroom*. The Modern Language Journal, 62(4), 175-182.

Rayner, S., \& Cantor, R. (1987). How fair is safe enough? The cultural approach to societal technology choice. Risk Analysis, 7, 3-9.

Renn, O. (1998). Three decades of risk research: accomplishments and new challenges. Journal of risk research, 1(1), 49-71.

Rosenthal, J. A. (1996). Qualitative descriptors of strength of association and effect size. Journal of Social Service Research, 21(4): 37-59.

Ross, I. (1975). Perceived Risk and Consumer Behavior: A Critical Review. In M.J. Schlinger (Ed.), Advances in Consumer Research, Volume 2. Association for Consumer Research, 1-20.

Rossi, P. H. (1951). The application of latent structure analysis to the study of social stratification. Unpublished Ph. D. dissertation, Columbia University. 
Rossi, P. H. (1979). 14. VIGNETTE ANALYSIS: UNCOVERING THE NORMATIVE STRUCTURE OF COMPLEX JUDGMENTS. Qualitative and quantitative social research: Papers in honor of Paul F. Lazarsfeld, 176.

Salganik, M. J. (2006). Variance estimation, design effects, and sample size calculations for respondent-driven sampling. Journal of Urban Health, 83(1), 98-112.

Salganik MJ, Heckathorn DD. (2004). Sampling and estimation in hidden populations using respondent-driven sampling. Sociol Method, 34, 193-239.

Scarfone, K, Souppaya, M., and Hoffman, P. (2011). Guide to Security for Full Virtualization Technologies. NIST: Special 800-125. http://csrc.nist.gov/publications/nistpubs/800-125/SP800-125-final.pdf (accessed April 15, 2014).

Schaeffer, N. C., \& Presser, S. (2003). The science of asking questions. Annual Review of Sociology, 65-88.

Schaeffer, N. C., \& Barker, K. (1995). Alternative methods of presenting bi-polar scales in television phone interviews: 1 to 7 vs -3 to +3 and neutral vs ambivalent. Annual Meeting Am. Assoc. Public Opin. Res., Fort Lauderdale, FL.

Schwarz, N., Hippler, H. J., Deutsch, B., \& Strack, F. (1985). Response scales: Effects of category range on reported behavior and comparative judgments. Public Opinion Quarterly, 49(3), 388-395.

Schwarz, N., Knäuper, B., Hippler, H. J., Noelle-Neumann, E., \& Clark, L. (1991). Rating scales numeric values may change the meaning of scale labels. Public Opinion Quarterly, 55(4), $570-582$.

Sjoberg, L. (2000). Factors in Risk Perception. Risk Analysis 20 (2000): 1-11.

Sosniak, L. A. (2006). Retrospective interviews in the study of expertise and expert performance. The Cambridge handbook of expertise and expert performance, 287-301.

Slatoff, W. J. (1970). With Respect to Readers: Dimensions of Literary Response. 
Sternberg, R. (2008). Cognitive psychology (Fifth Ed.) Cengage Learning.

Tarr, M. J. (1995). Rotating objects to recognize them: A case study on the role of viewpoint dependency in the recognition of three-dimensional objects. Psychonomic Bulletin \& Review, $2(1), 55-82$.

Tarr, M. J. (2000). Visual pattern recognition. Encyclopedia of psychology, 1-4.

US GAO 04-376. 2004. Agencies need to implement consistent processes in authorizing systems for operation. Report from The US Government Accountability Office.

van Eijnatten, F. M., van der Ark, L. A., \& Holloway, S. S. (2014). Ipsative measurement and the analysis of organizational values: an alternative approach for data analysis. Quality \& Quantity, 1-21.

Verdon, D., \& McGraw, G. (2004). Risk analysis in software design. IEEE Security and Privacy. Mag. 2(4), 79-84.

Wang, L, Jajodia, S., Singhal, A., Cheng, P., \& Noel, S. (2014). k-Zero Day Safety: A Network Security Metric for Measuring the Risk of Unknown Vulnerabilities. IEEE Transactions on Dependable and Secure Computing, 11(1), 30-44.

Weber, E. U., Blais, A. R. E., \& Betz, N. E. (2002). A Domain-specific Risk-attitude Scale: Measuring Risk Perceptions and Risk Behaviors. Journal of Behavioral Decision Making, 15, 263-290.

Wickens, C. D., \& Carswell, C. M. (1997). Information processing. Handbook of human factors and ergonomics, 2, 89-122.

Zhang, Y., Dao, S.K., Vin, H., Alvisi, L., \& Lee, W. (2002). Heterogeneous Networking: A New Survivability Paradigm. IEEE NSPW'01.New Mexico, USA: Cloudcroft. September 10-13, pp. 33-39.

Zhang, S., Ou, X.,Singhal, A., \&Homer, J. (2011). An empirical study of a vulnerability metric aggregation method. In Arabnia, H.R., M.R. Grimaila, G. Markowsky, \& S. Aissi (Eds.), Proceedings of the 2011 International Conference on Security \& Management (SAM 2011). 
WORLDCOMP'11. Las Vegas, Nevada: CSREA Press, July 18-21, 2011. http://www.worldcomp-proceedings.com/proc/proc2011/sam/contents-vol-i.pdf 


\title{
Effect of Network Infrastructure Factors on Information System Risk Judgments Jennifer A. Cowley, Frank L. Greitzer, Bronwyn Woods
}

\author{
Figure Captions
}

Figure 1. Network Topology Legend

Figure 2. Network Topologies for Scenarios 1-16

Figure 3. Overview of Survey Responses by Participant Number

Figure 4. Boxplots of the Risk Rating Data by Scenario.

Figure 5. Bar plots (frequencies) of the Risk Ranking Data by Scenario.

Figure 6. Histogram of participant-specific estimated intercept terms in linear model. 